\title{
Decoded: A Case Report on Dedifferentiated Liposarcoma on the Gluteal Area
}

\author{
Juan Paolo David S. Villena, MD, ${ }^{1}$ Eileen Liesl A. Cubillan, MD $^{1}$ and Ann Camille Q. Yuga, MD² \\ ${ }^{1}$ Department of Dermatology, Philippine General Hospital, University of the Philippines Manila \\ ${ }^{2}$ Department of Surgery, Philippine General Hospital, University of the Philippines Manila
}

\begin{abstract}
Dedifferentiated liposarcoma is a soft tissue sarcoma of adipocytic lineage. Histopathology and immunohistochemistry are essential for diagnosis. A 51-year-old Filipino woman presented with a rapidly enlarging left gluteal tumor. Histopathology revealed a multilobulated tumor having prominent myxoid stroma with numerous stellate-shaped, atypical cells bearing atypical mitotic figures. Other lobules were composed of sheets of pleomorphic cells, with atypical mitotic figures. The tumor stained positively with alcian blue, vimentin, MDM2 and p16 stains. Other immunohistochemical (IHC) studies done (pancytokeratin, CK7, CK 20, CD 34, CEA, desmin, EMA, SMA, S100) showed negative results. After a $2 \mathrm{~cm}$ wide excision of the sarcoma, patient was free from local tumor recurrence for 2 months, after which she was lost to follow-up. We report this case and a brief review of the current literature on dedifferentiated liposarcoma.
\end{abstract}

Key Words: dedifferentiated liposarcoma, immunohistochemistry, MDM2, p16

\section{INTRODUCTION}

Dedifferentiated liposarcoma (DDLS) is a biphasic neoplasm with two components: an atypical lipomatous tumor and an area consistent with a high-grade sarcoma. ${ }^{1,2}$ It is usually seen in elderly populations and is known to have a slight male predilection. ${ }^{3}$ Commonly found in the retroperitoneum but rarely in the legs and buttocks, it comprises only $2 \%$ of all known DDLS cases. ${ }^{4}$ The reported incidence of retroperitoneal DDLS is $20.9 \% ;^{5}$ however, incidence for DDLS of the gluteal area is unknown. In the Philippines, there is no data on the incidence of DDLS; only one case report was available upon literature search. The case report described another case of DDLS diagnosed via peritoneal biopsy in a middle-aged Filipino woman who was previously diagnosed with tuberculosis, and who succumbed to multi-organ failure. ${ }^{6}$ In this report, we present a case of DDLS in the gluteal area of a middle-aged Filipino woman as well as a brief review of the current literature on DDLS.

\section{CASE DESCRIPTION}

E-Poster presented in the $25^{\text {th }}$ Annual PGH Research Week 2020 on November 16-20, 2020, at the University of the Philippines Philippine General Hospital.

Corresponding author: Juan Paolo David S. Villena, MD Department of Dermatology Philippine General Hospital

University of the Philippines Manila

Email: jsvillena1@up.edu.ph
This is a case a 51-year-old Filipino woman with a 2-month history of an ulcerated tumor on the left gluteal area. The lesion began as an aggressively enlarging painless pustule that spontaneously ulcerated. No other signs and symptoms were noted. Its progressive enlargement and spontaneous bleeding prompted consult at the Department of Dermatology in the Philippine General Hospital. On examination, there was a $10 \times 8.8 \times 2 \mathrm{~cm}$ solitary, ulcerated, 

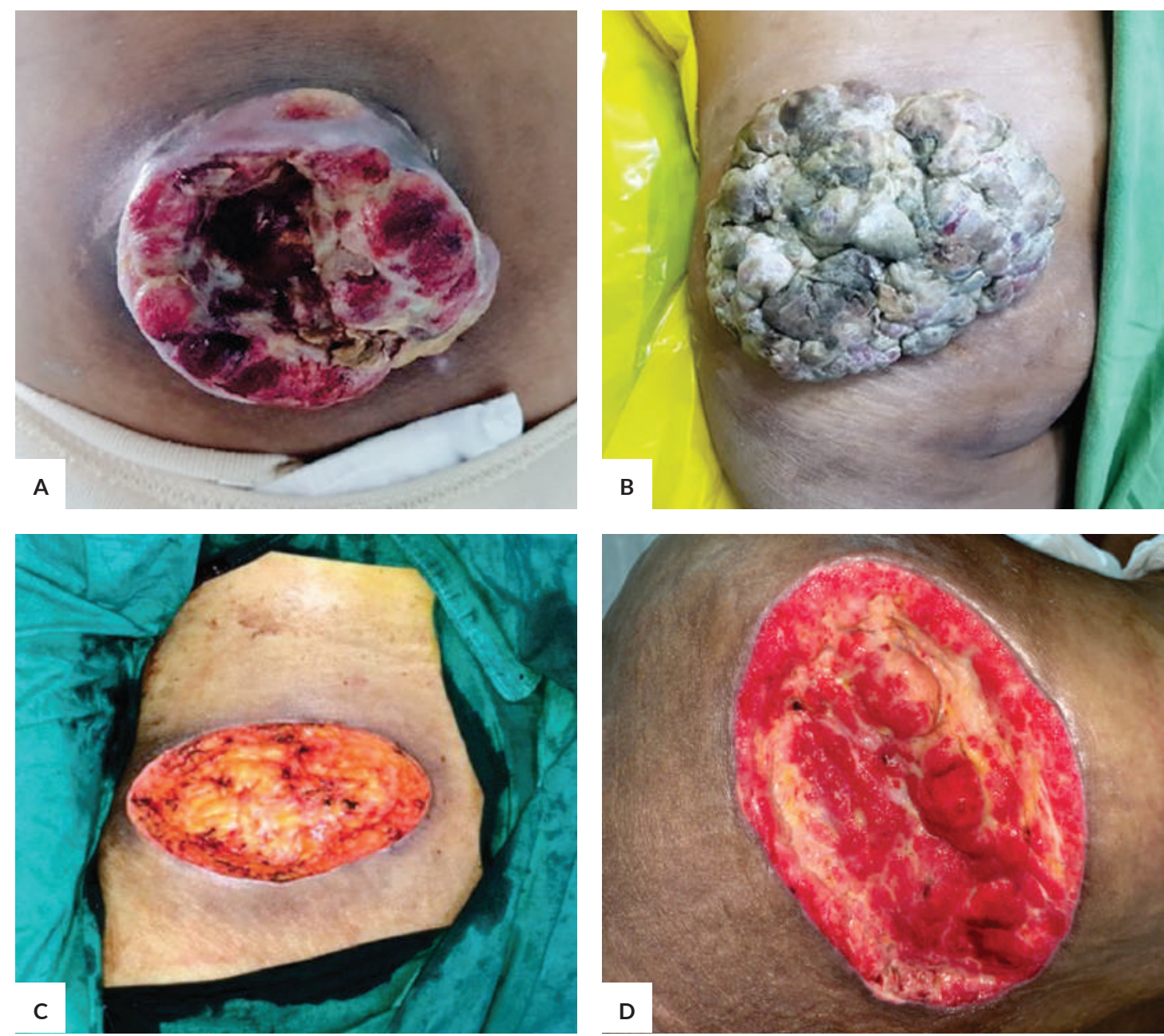

Figure 1. Left gluteal tumor. (A) Initial size of left gluteal tumor measuring $10 \times 8.8 \times 2 \mathrm{~cm}$. (B) Gluteal tumor grew to a size of $16 \times 12 \times 6 \mathrm{~cm}$ two months from initial consult. (C) Wide excision of tumor, intraoperative. (D) One week post-wide excision.

friable tumor with hemorrhagic crusting and blood clots over the left gluteal area (Figure 1A). Patient was afebrile and had no palpable lymphadenopathies over the inguinal, axillary and cervical areas during this initial consult. The rest of the systemic examination findings were unremarkable.

An incisional biopsy was done on this tumor which showed a multilobulated specimen whose lobules were separated by fibrous septae (Figure 2A and 2B). The tumor lobules had different characteristics. Some lobules had stellate cells with prominent pleomorphism and mitotic figures (10-15 per high-power field) laid on a myxoid stroma (Figure 2C and 2D) while other lobules contained solid sheets of highly pleomorphic cells and numerous mitotic figures (20-25 per high-power field) (Figure 2E). Some cells were multivacuolated, having an indented nucleus with darkly staining nucleoli, and were initially assessed to be pseudolipoblasts (Figure 2F). The histologic findings were consistent with a soft tissue sarcoma, with a diagnosis favoring myxofibrosarcoma (MFS), pending immunohistochemical examination. A computed tomography $(\mathrm{CT})$ scan was also requested to assess tumor depth and characteristics.

The patient was lost to follow-up and came back after two months presenting with an exophytic, tan-colored tumor measuring $16 \times 12 \times 6 \mathrm{~cm}$ which had a foul-smelling yellow, purulent discharge (Figure 1B). During this time, the patient also presented with pallor, generalized malaise and weakness, with undocumented weight loss, as well as bipedal edema and palpable left inguinal lymph nodes. CT scan showed an exophytic, hypodense mass lesion measuring $13.1 \times 15.2 \times 4.2 \mathrm{~cm}$ with prominent left sided lymphatics. There was no muscular or osseous involvement noted. She was then brought to the emergency department due to pallor and generalized weakness. While admitted in the hospital, biopsy findings were reviewed. The cytomorphology of the previously identified pseudolipoblasts were also consistent with that of lipoblasts (Figure $2 \mathrm{~F}$ ); hence, liposarcoma was considered. Additional work-ups were done, including the special stains and immunohistochemical studies on her 

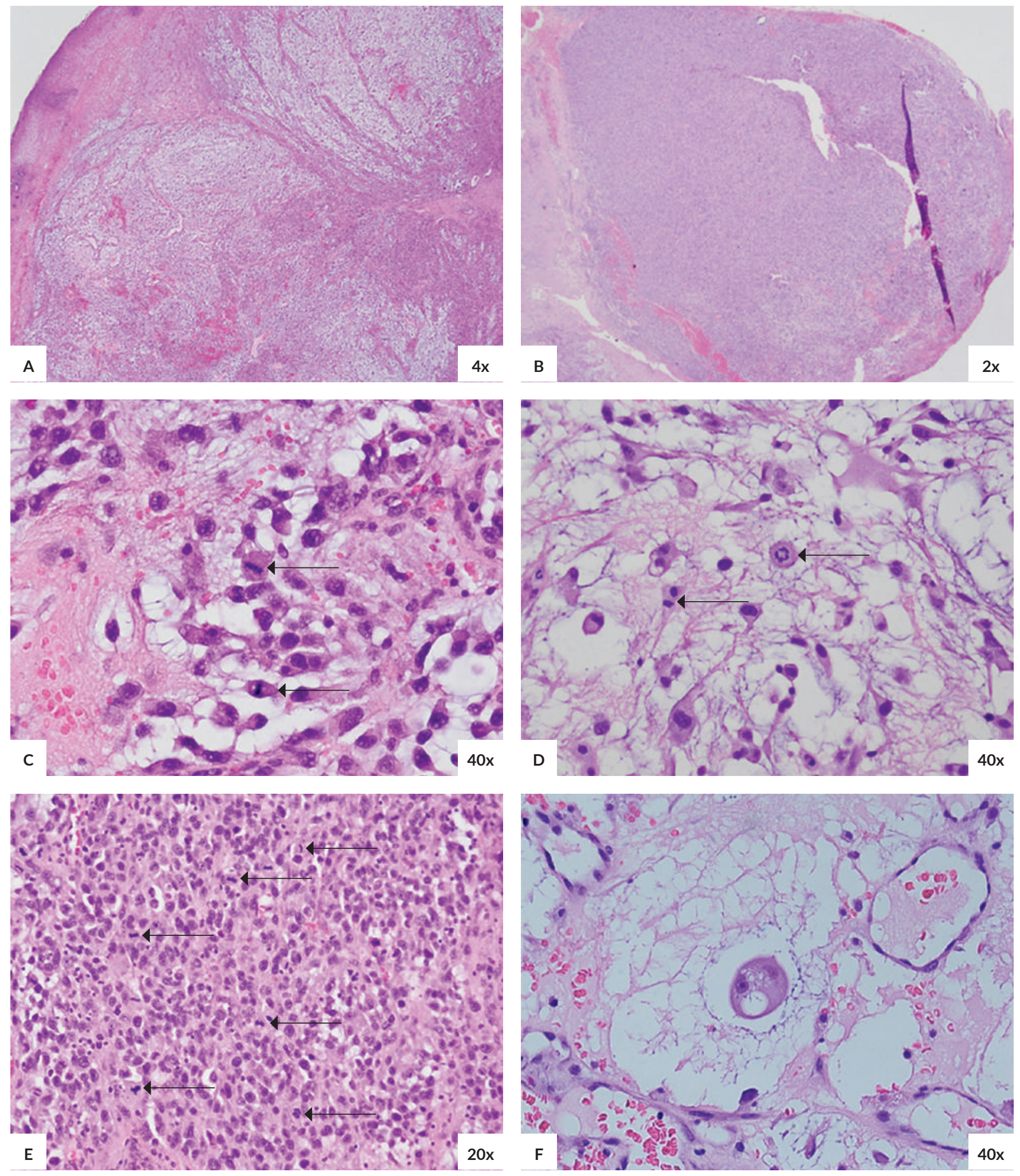

Figure 2. Histopathologic findings of gluteal tumor (hematoxylin and eosin). Biopsy showed a multilobulated specimen separated by fibrous septa. Some lobules were composed of stellate cells laid on a prominent myxoid stroma (A, 4x) while other lobules contained solid sheets of highly pleomorphic cells with epithelioid features (B, 2x). Cells had atypical nuclei and numerous mitotic figures as pointed by the black arrows (C, 40x; D, 40x; E, 20x). Lipoblasts were also appreciated as multivacuolated cells having an indented nucleus with darkly staining nucleoli (F, 40x). 

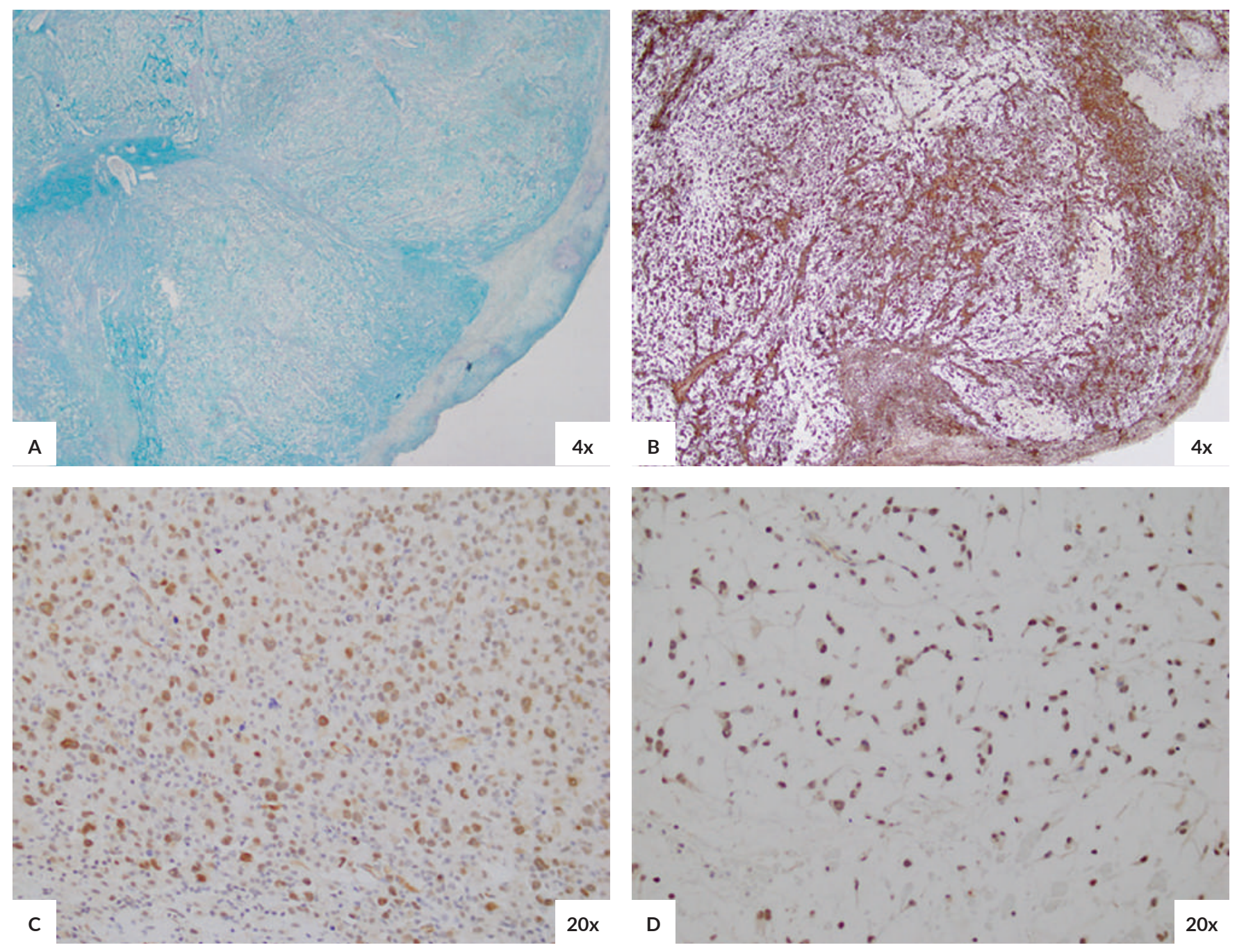

Figure 3. Immunohistochemical profile of gluteal tumor. There was diffuse staining with alcian blue (A, 4x). Vimentin showed strong positive staining as well (B, 4x). MDM2 staining showed strongly positive staining of the atypical cells (C, 20x). P16 also showed strong nuclear staining of the atypical cells (D, 20x).

previous biopsy, which showed positive staining for alcian blue (Figure 3A), vimentin (Figure 3B), MDM2 (Figure 3C) and p16 (Figure 3D). Staining for cytokeratin 7 and 20 (CK7 and CK20), CD34, carcinoembryonic antigen (CEA), epithelial membrane antigen (EMA), desmin, pancytokeratin, smooth muscle actin (SMA), and S-100 was negative. This led to the diagnosis of DDLS (Figure 4).

CT scan of the chest revealed no pulmonary metastatic lesions. The patient was assessed to be at stage IV, since the tumor measured greater than $15 \mathrm{~cm}$ in its greatest dimension, and presented with regional lymph node metastasis despite no evidence of distant organ metastasis. The General Surgery Service performed excision of the inguinal node and wide excision of the left gluteal tumor with at least $2 \mathrm{~cm}$ margin. (Figure 1C and 1D). As the tumor was infected, the plan was to do a delayed reconstruction once tissue culture showed negative microbial growth. An immediate reconstruction with split thickness skin grafting on an infected wound bed may lead to complications such as infection and graft loss. Hence, the wound bed was covered temporarily with hydrophilic dressing. Postoperatively, intravenous antibiotics was continued while waiting for the tissue culture results. Unfortunately, the patient had status asthmatics and went into cardiac arrest. She was revived after advanced cardiac life support was rendered. However, because of the untoward events in the course of her condition, her family decided not to proceed with the second surgery with the plan of reconstruction and instead healing was done by secondary intention and application of silver-based dressing. There was also no consent to have further adjuvant treatment such as radiation therapy. The patient was advised to follow-up monthly for the first 3 months. She was noted to be free from local tumor recurrence during the first 2 months. However, was eventually lost to follow-up thereafter. 


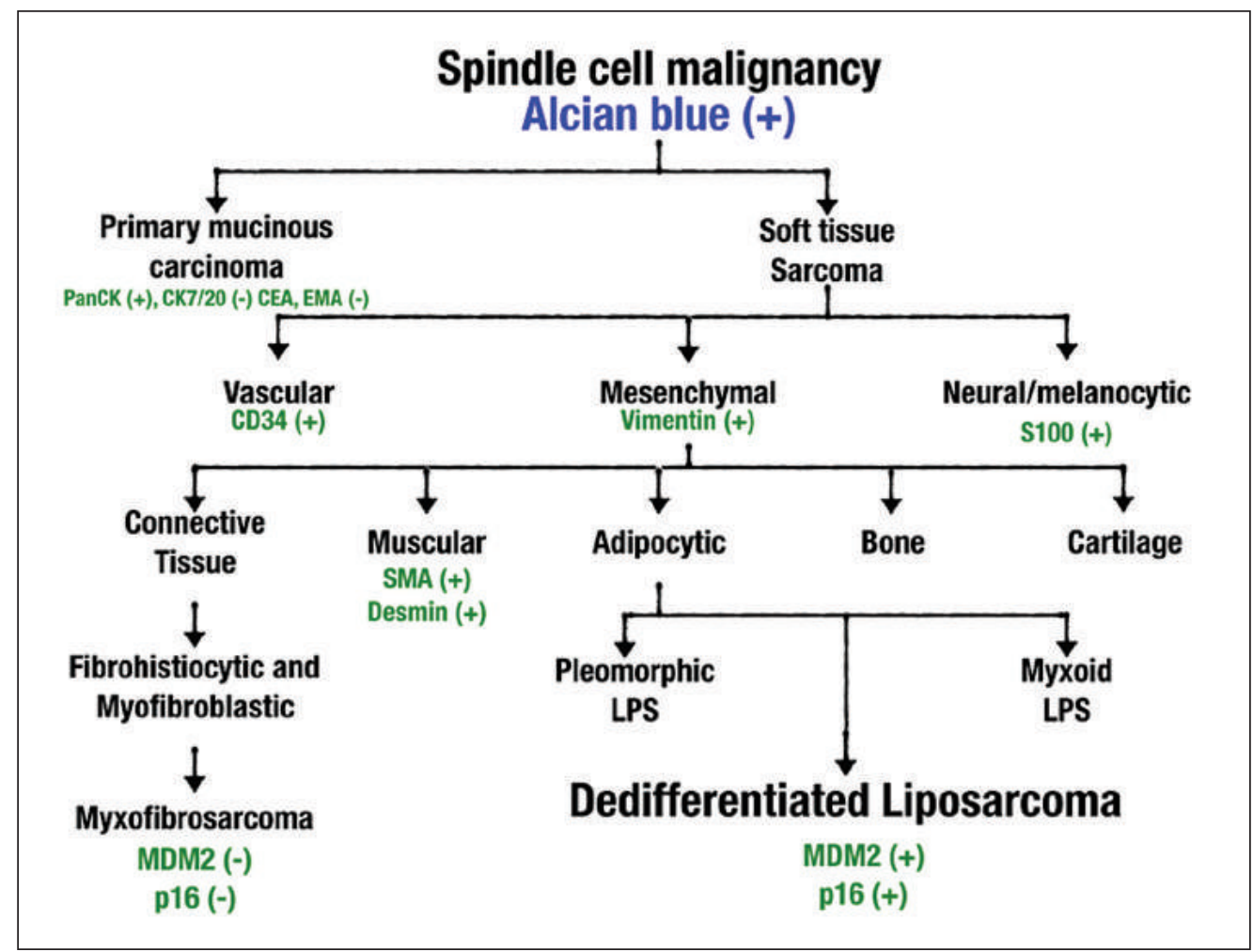

Figure 4. Flowchart of immunohistochemical stains done on left gluteal tumor specimen. Close histologic differentials for a prominent myxoid stroma with a spindle cell malignancy include primary mucinous carcinoma and soft tissue sarcomas. Soft tissue sarcomas, having spindled cell morphology, are classified according to their embryonic origin or cell lineage. Vascular tumors stain positive for CD34, neural tumors for S-100 and mesenchymal tumors for vimentin. In the case of mesenchymal tumors, further IHC studies are needed to definitively identify the cell lineage of the tumor.

\section{DISCUSSION}

The liposarcomas (LPS) are malignant tumors with adipocytic differentiation. Their incidence is at 2.5 per million $^{7}$ and comprise $25 \%$ of all soft tissue sarcomas, ${ }^{8}$ making them the most common soft tissue sarcoma found in adults. ${ }^{7}$ Liposarcomas, in general, arise de novo, and not from malignant degeneration of previously benign adipocytic tumors such as a lipoma. ${ }^{4}$ The most common type of LPS is the spectrum of well-differentiated and DDLS. DDLS are biphasic neoplasms composed of a welldifferentiated LPS and a second component of a high-grade pleomorphic sarcoma usually seen as nonlipogenic areas of atypia adjacent to the well-differentiated component. ${ }^{1,2}$ It is usually seen in elderly populations and has a slight male predilection. ${ }^{3}$ It is commonly found in the retroperitoneum and rarely appears in the legs and buttocks, composing only $2 \%$ of all DDLS. Dedifferentiation is common in deep-seated tumors (such as that in the retroperitoneum, spermatic cord and mediastinum) but is remarkably rare in subcutaneous lesions that are usually seen in the trunk and legs, particularly the thigh., ${ }^{2,9}$
DDLS is characterized by a supernumerary ring or giant marker chromosome that may produce overexpression of MDM2 and CDK4 due to amplification in the q13-15 region of chromosome $12 ;{ }^{9-11}$ hence, the positivity of MDM2 and CDK4 staining. Other types of liposarcomas are the myxoid type and the pleomorphic type. The myxoid or round cell type of LPS is characterized by a specific translocation between chromosomes 12 and 16 and does not cause over expression of the MDM2 and CDK4. ${ }^{10}$ Pleomorphic liposarcoma, the least common subtype, has a complex genomic profile with numerous gains and losses, bearing no specific immunohistochemical blueprint that can be adapted. ${ }^{10}$

Routine biopsy, particularly incisional or wedge biopsy, is essential for diagnosis. ${ }^{12}$ This is to visualize the abrupt transition from conventional well-differentiated liposarcoma to a dedifferentiated component, with its numerous atypical cells, numerous atypical mitotic figures and abundant myxoid stroma. Due to the abundant rich stroma in which these lesions are embedded, DDLS may closely mimic myxofibrosarcoma and can be an important histologic differential diagnosis. 
Prior to performing the IHC studies, myxofibrosarcoma was the primary consideration in this patient because of the location of the tumor which was closely related to the extremity as well as the dense mucinous stroma as well as multiple mitotic figures seen on histopathology. There were seemingly elongated and arcuate vessels in the tumor as well, which are highly characteristic for myxofibrosarcoma. Another close histologic differential diagnosis of this case was primary mucinous stroma, owing to the myxoid background where the tumor was embedded. At this point, the primary service was not highly considering DDLS as most histopathologic studies would have revealed an abrupt transition from a well-formed adipose tissue to a dedifferentiated component; which was not present in this case. This may be due to the partial, relatively superficial biopsy sampling obtained from the first incisional biopsy. It is important to note that both clinically and histologically, many soft tissue sarcomas closely mimic one another. This highlights the important role of IHC in the identification of their cell lineage markers that will confirm the histologic diagnosis these tumors in the absence of amplification and gene studies. Upon performance of IHCs, tissue samples of the first biopsy were reviewed by multiple pathologists and a few lipoblasts were seen on closer examination. These lipoblasts are multivacuolated cells having an indented nucleus with darkly staining nucleoli and is seen in a proportion of patients with DDLS. This significant finding prompted to consider and work up the patient for the diagnosis of DDLS.

Diffuse nuclear staining with MDM2 and CDK4 is an IHC profile that may point to the diagnosis of DDLS over MFS and other soft tissue sarcomas., ${ }^{5,9}$ Positive staining with vimentin is expected in DDLS because these are tumors of soft-tissue lineage. Alcian blue staining will also yield positive results as DDLS are usually embedded in a myxoid or mucin-rich stroma. In a study by Kammerer-Jacquet, they suggested the use of $\mathrm{p} 16$ as another surrogate marker in the diagnosis of DDLS..$^{13}$ In their study, they concluded that the sensitivity and specificity of using p16 alone in diagnosing DDLS was $94.4 \%$ and $70 \%$ respectively; however, when used in combination with MDM2, sensitivity remains at $94.4 \%$ and specificity increases to $95 \% \cdot{ }^{13}$ Specificity further improves to $100 \%$ when all three markers, MDM2, CDK4 and p16 are used altogether. ${ }^{13}$ CDK4 however, was not done in our patient due to unavailability in our country. In some cases, pleomorphic areas may express desmin, actin or $\mathrm{CD} 34^{11}$ which make leiomyosarcoma a differential diagnosis. However, leiomyosarcoma is negative for MDM2/ CDK4. ${ }^{11}$ Newer molecular diagnostic techniques such as fluorescence in situ hybridization (FISH) and reverse transcriptase-polymerase chain reaction (RT-PCR) tests are considered important tools that may also be used in the diagnosis of DDLS and other soft tissue sarcomas. ${ }^{12}$

Further work-up such as radiographic assessment is important in patients with DDLS because it allows the clinician to determine the extent of the primary tumor, aids the surgeon in surgical planning and allows assessment for metastatic disease. ${ }^{12}$ Magnetic resonance imaging (MRI) is the preferred modality for all soft tissue sarcomas found in the head and neck, trunk and extremities while computed tomography (CT) scan is a better tool in retroperitoneal and visceral sarcomas. ${ }^{13}$ In the diagnostic work-up, CT scan of the chest is the gold standard in assessing the lungs to search for metastasis. ${ }^{12}$ In our patient, a pelvic CT scan was ordered and showed an exophytic hypodense mass lesion measuring $13.1 \times 15.2 \times 4.2 \mathrm{~cm}$ with prominent left sided lymphatics. There was no muscular or osseous involvement noted.

The American Joint Committee on Cancer (AJCC) $\left(8^{\text {th }}\right.$ ed) proposed a 4-tier staging system that aids in prognostication and surgical planning of soft tissue sarcomas. The AJCC staging system is based on histologic grade, tumor size $(\mathrm{T})$, nodal involvement $(\mathrm{N})$ and distant metastasis $(\mathrm{M})$. Histologic grading of soft tissue sarcomas is based on total differentiation, mitotic count and necrosis score. ${ }^{12}$ Total differentiation is graded based on how aggressive the tumor is. Tumors may be notoriously aggressive, such as DDLS or less aggressive, such as MFS. Mitotic count refers to the number of atypical mitotic figures visualized per high-power field. Necrosis is graded based on the percentage of necrotic material present in the tumor on histologic examination. Presence of more poorly differentiated tissues, more atypical mitotic figures and higher percentage of necrosis pertains to a more advanced grade. DDLS is given a score of 3 for total differentiation due to its very aggressive behavior while MFS is assigned a score of 2 because of its relatively benign course. In our case, more than $50 \%$ necrosis was seen on gross examination of the tumor (score $=2$ ) and multiple mitotic figures of more than 20 per high-power field were visualized (score $=3$ ). A cumulative score of 8 for the three aforementioned parameters corresponds to a grade 3 tumor, indicating a high-grade neoplasm. ${ }^{12}$ Based on the AJCC staging system, patient was assessed to be stage IV since the tumor measured greater than $15 \mathrm{~cm}$ in greatest dimension (T4) and presented with regional lymph node metastasis (N1) despite no evidence of distant organ metastasis (M0).

A multidisciplinary approach is recommended in managing patients with soft tissue sarcomas. The current standard of care for all soft tissue sarcomas of the extremities, whether MFS or DDLS, remains to be surgical management. ${ }^{12,14}$ Wide resection ${ }^{4}$ with tumor-free margins of at least $1 \mathrm{~cm}$ is the recommended procedure as this reduces the risk of local recurrence. ${ }^{14}$ Based on the National Comprehensive Cancer Network (NCCN) guidelines, for stage 4 soft tissue sarcomas of the extremities, tumor management via surgical excision is the treatment of choice. ${ }^{15}$ Pre-operative tumor debulking using 50-Gray external beam radiotherapy ${ }^{16}$ has been reported to reduce the rates of local recurrence. ${ }^{13}$ In some studies, disease control may be improved with the use of systemic chemotherapy, particularly in LPS of 
the extremities. ${ }^{16}$ Doxorubicin, ifosfamide, gemcitabine and docetaxel are recommended as possible first-line systemic chemotherapeutic agents for DDLS. ${ }^{16}$ Novel second-line antineoplastics such as trabectidin, eribulin, palbociclib are ongoing clinical research. ${ }^{16}$

Wide excision of the tumor with at least $2-\mathrm{cm}$ margins was performed by the General Surgery service. Lymph nodes were also excised and it showed a high-grade malignant neoplasm with mucinous features, consistent with DDLS. The patient might also benefit in receiving adjuvant radiation therapy for a better local control. However, because of the complicated postoperative course of the patient and potential delay in the wound healing, radiation therapy, which is usually 4 weeks post-operatively, was not given immediately.

DDLS is notorious for having a high rate of local recurrence especially when found in deeper areas such as the retroperitoneum ${ }^{1}$ and bears a metastatic risk of $15-20 \%{ }^{3}$ A more worrisome histologic grade (i.e., grade 3) may indicate risk for developing distant metastasis most often seen in the lung and bones. ${ }^{3}$ Local recurrence, on the other hand, is brought about by an infiltrative growth pattern, signified by extension along vascular and fascial planes; therefore, clearance in the surgical margins determines the risk for developing local recurrence. ${ }^{9}$ DDLS lesions on the extremities have a better 5-year survival rate because they rarely develop local recurrence and distant metastasis, compared to lesions on the retroperitoneum. ${ }^{15}$ This may be explained by the increased difficulty in removing the entire tumor and getting tumor-free margins in deep-seated lesions of the retroperitoneum..$^{15}$ In this case, the wide excision by General Surgery Service yielded negative tumor margins, indicating a better prognosis in terms of local recurrence. However, the presence of tumor spread to the regional lymph nodes automatically upstages the patient to stage IV, hence a poorer prognosis. The patient followed up two months after surgery and was noted to be free from local tumor recurrence. However, the patient has since been lost to follow-up.

\section{CONCLUSION}

It is a challenge to diagnose soft tissue sarcomas with only routine staining, especially if they present in unusual locations. Early recognition of the tumors and maximizing the diagnostic tools are essential for accurate diagnosis especially in aggressive tumors, such as DDLS. This case emphasizes the importance of IHCs especially in soft tissue sarcomas as these can identify the lineage of the tumor and can aid in prognosticating the patients. Lastly, a multidisciplinary approach in rare cases like this is fundamental in providing prompt and appropriate management.

\section{Statement of Authorship}

All authors participated in data collection and manuscript writing, and approved the final version submitted.

\section{Author Disclosure}

All authors declared no conflicts of interest.

\section{Funding Source}

This study was self-funded by the authors.

\section{REFERENCES}

1. Brenn, T. Fitzpatricks Dermatology in General Medicine. 8th ed. New York: McGraw-Hill; c2012. Chapter 129, Neoplasms of Subcutaneous Fat; p. 1489-1498.

2. Calonje E, Brenn T, Lazar, A. Mckee's Pathology of the Skin. 4th ed. Amsterdam: Elsevier-Saunders; c2012. Chapter 35, Connective tissue tumors; p. 1600-1603.

3. Sioletic S, Dal Cin P, Fletcher CDM, et al. Well-differentiated and dedifferentiated liposarcomas with prominent myxoid stroma: analysis of 56 cases: Liposarcomas with myxoid stroma. Histopathology. 2013;62:287-93.

4. Kaddu S. Dermatology. 4th ed. Amsterdam: Elsevier; c2018. Chapter 117, Smooth muscle, adipose and cartilage neoplasms; p. 2097-9.

5. Wei S, Henderson-Jackson E, Qian X, et al. Soft Tissue Tumor Immunohistochemistry Update: Illustrative Examples of Diagnostic Pearls to Avoid Pitfalls. Archives of Pathology \& Laboratory Medicine. 2017;141:1072-1091.

6. Montejo YBE. Dedifferentiated Liposarcoma Masquerading as Tuberculosis in a 25-year-old Female Patient. Philippine Journal of Internal Medicine. 2016;54.3.1-4.

7. Schwartz RA, Centurion SA. Liposarcoma [Internet]. [cited 2020Sep13]. Background, Pathophysiology, Etiology. Medscape; 2020. Available from: https://emedicine.medscape.com/article/1102007overview

8. Lee ATJ, Thway K, Huang PH, et al. Clinical and Molecular Spectrum of Liposarcoma. JCO. 2018;36:151-9.

9. de Saint Aubain N. Pleomorphic cell tumors of soft tissue: Differential diagnosis [Internet]. [cited 2020 Sep13]. Lecture presented at: Forums of Pathology; 2019 Feb 25. Available from: http://www.forpath.org/ Workshops/minutes/0401/Pleomorphic\%20cell\%20tumors\%20 of $\% 20$ soft $\% 20$ tissue.pdf

10. Coindre J-M, Pédeutour F, Aurias A. Well-differentiated and dedifferentiated liposarcomas. Virchows Archiv. 2010;456:167-179.

11. Fisher C. Immunohistochemistry in diagnosis of soft tissue tumours: Soft tissue tumour immunohistochemistry. Histopathology. 2011;58:1001-1012.

12. Ryan, CW, Meyer J, Maki R, Pollock RE, Savarese DMF. Clinical presentation, histopathology, diagnostic evaluation, and staging of soft tissue sarcoma. Up To Date. Massachusetts: 2012.

13. Kammerer-Jacquet S-F, Thierry S, Cabillic F, et al. Differential diagnosis of atypical lipomatous tumor/well-differentiated liposarcoma and dedifferentiated liposarcoma: utility of p16 in combination with MDM2 and CDK4 immunohistochemistry. Human Pathology. 2017;59:34-40.

14. Gebhardt MC, Pollock RE, Maki R, Collins KA, Savarese DMF. Surgical resection of primary soft tissue sarcoma of the extremities. Up To Date. Massachusetts: 2018.

15. NCCN Guidelines for Soft Tissue Sarcoma Patients. Version 2. [Internet] 2018 [cited 2019 February 4]. Available from: https://www. nccn.org/patients/guidelines/sarcoma/4/.

16. Nassif NA, Tseng W, Borges C, et al. Recent advances in the management of liposarcoma. F1000Research. 2016;5:2907. 